\title{
Detox your Life
}

Wie Sie Ihren Körper beim Entgiften unterstützen und sich von Belastungen befreien. R. Höhl

Wir sprachen mit Dr. Christian Matthai über sein jüngstes Buch

An welche Zielgruppen wendet sich Ihr neues Werk?

MATTHAI: An alle Menschen, die et was für ihre Gesundheit tun wollen sich von Belastungen befreien und ih individuelles gesundes Gleichgewicht wieder herstellen möchten.

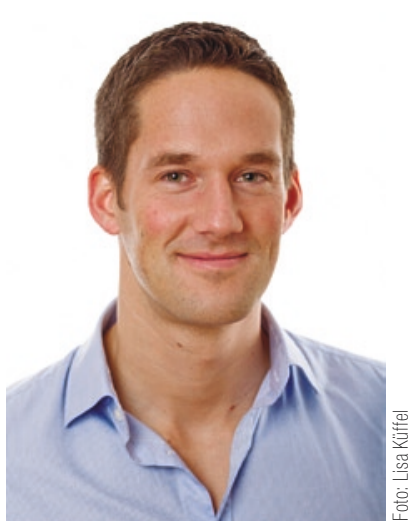

Dr. Christian Matthai

ist Gynäkologe und Lifestyle Coach be "woman \& health". Er ist ferner Autor

Wussenchationen sowie Ernährung Sport Orthomolekulare Medizin.

Was ist die Hauptaussage Ihre Buches?

MATTHAI: Es gibt eine Menge Dinge die man im Lebensalltag besonders oft tun und welche, die man tunlichs meiden sollte. Bei „Detox your Life handelt es sich um einen LifestyleRatgeber, der genau erklärt, was jede von uns tun kann, um dem Körpe beim Entgiften zu helfen, sich von Belastungen $\mathrm{zu}$ befreien und das Immunsystem optimal zu unterstützen.

Was fällt aus medizinischer Sich unter den Terminus „Entgiftung“? MATTHAI: Beim Entgiften habe ich mich auf unsere beiden Haupt-Entgiftungsorgane Leber und Nieren konzentriert. Entgiften bedeutet einerseits, belastende Substanzen wi Alkohol oder Medikamente über die Leber abzubauen und zu verstoffwechseln und andererseits, über die Nieren alle harnpflichtigen Stoffe auszuscheiden. Und diese Organe kann man eben ganz gezielt, z. B. über die Ernährung, bei ihren Aufgaben unterstützen.

Welche Methoden der Entgiftung beschreibt das Buch?

MATTHAI: Es geht dabei im Großen und Ganzen um Lebensstilinterventionen. Den Schwerpunkt des Buches bildet das Thema Ernährung.

Die Prävalenz der Adipositas steig weltweit und stellt bereits für Kinder ein Problem dar; Herz-Kreislauferkrankungen und Karzinome führen in der Liste der Morbiditätsund Mortalitätsursachen. Wir Ärzte

Dr. Renate Höhl

ist Ärztin für Allgemeinmedizin und arbeite seit 1998 als wissenschaftliche Fachredakteurin beim Springer Verlag Wien, lettende wissen aber grundsätzlich (und seit langem), dass wir diesen Problemen präventiv begegnen müssen. Was läuft Ihrer Meinung nach in de heutigen Gesellschaft falsch? MATTHAI: Primär müssen wir den Menschen klar machen, dass jede einzelne jeden Tag die Chance hat, seinen eigenen Beitrag zur Gesunderhaltung zu leisten - eben über den richtigen Lebensstil. Die Menschen müssen realisieren, dass es kein $\mathrm{Lu}-$ xus, sondern eine absolute Notwendigkeit ist, sich um seine Gesundheit zu bemühen, indem wir uns bewegen und gesund ernähren.

Welche Tipps geben Sie den Lesern Ihres Buches?

MATTHAI: Die Leser erfahren, was sie möglichst oft in ihren Speiseplan einbauen sollen, welche Wirkungen diverse Lebensmittel auf unsere $\mathrm{Ge}$ sundheit haben oder was uns belasten und unsere Gesundheit gefährden kann. Die Leser erlernen, wie man das Immunsystem optimal unterstützt. Untermalt wird das Ganze durch eigens von mir kreierte $\mathrm{Re}-$ zepte.

Die meisten Diäten sind aufwändig und wenig alltagstauglich. Wie sieh es mit Ihrem Ernährungskonzept aus?

MATTHAI: Was mir immer sehr wichtig ist, sind drei Komponenten: Leicht gesund und einfach zuzubereiten muss es sein. Wenn man gerne ein paar Kilos abnehmen möchte, dann sollte man auf Zwischenmahlzeiten verzichten und abends die Kohlenhydrate meiden

Wie stehen Sie zu Nahrungsergänzungsmitteln?

MATTHAI: Die orthomolekulare Me dizin ist ein sehr interessantes Thema. Um in diesem Bereich sinnvoll arbeiten zu können, muss man sich intensiv mit der vorliegenden Literatur beschäftigen. Es gibt viele Substanzen die man überaus sinnvoll sowohl im präventiven, als auch im therapeutischen Bereich einsetzen kann. Es is aber sich nicht alles gut, was Nahrungsergänzungsmittel ist. Da muss man sehr genau unterscheiden und auch richtig dosieren. Auch über den Einsatz von Pflanzenextrakten sollte man nachdenken, da diese sehr gesund sind und man möglicherweise nicht alles Gesunde immer zu essen bekommt.

Ihr Buch enthält wunderschöne Bilder. Wurden die Fotos eigens für das Buch aufgenommen?

MATTHAI: Ja, die Speisen wurden von mir kreiert, am Tag des Shootings gemeinsam mit meiner Verlobten Lisa zubereitet und von einem Fotografen (Peter Barci) eigens für das Buch abgelichtet. Wir sind an diesem Tag 12 Stunden in der Küche gestanden und freuen uns sehr, dass Ihnen die Bilder gefallen!

Erlauben Sie uns einen Blick in die Zukunft: Haben Sie Pläne für ein weiteres Werk?

MATTHAI: Kurz nach dem Erscheinen von „Detox your Life“" das ja nur
12 Monate nach meinem letzten Buch Change - das Lifestyle-Abnehmprogramm" veröffentlicht wurde, habe ich mir gedacht, dass ich jetzt mal ein bisschen Pause mache. Kurz darau hat es mich aber schon wieder in den Fingerspitzen gejuckt. Viele Lese und Patienten fragen nach mehr Rezepten. Diesem Wunsch gehe ich nach und bin bereits bei einem neuen Buchprojekt, das in die leichte DetoxKochbuch- Richtung geht. Ich bin und bleibe eben „Dr. Cook“.

Herzlichen Dank für das interessante Gespräch!

\section{Dr. Christian Mattha}

Detox your Life

Wie Sie Ihren Körper beim Entgiften Wen befreien

144 Seiten, durchgehend farbig ISBN 978-3-7088-0479-8 EUR 17,95 / CHF 31,50 1. Auflage, Februar 2010 政 www.kneippverlag.com

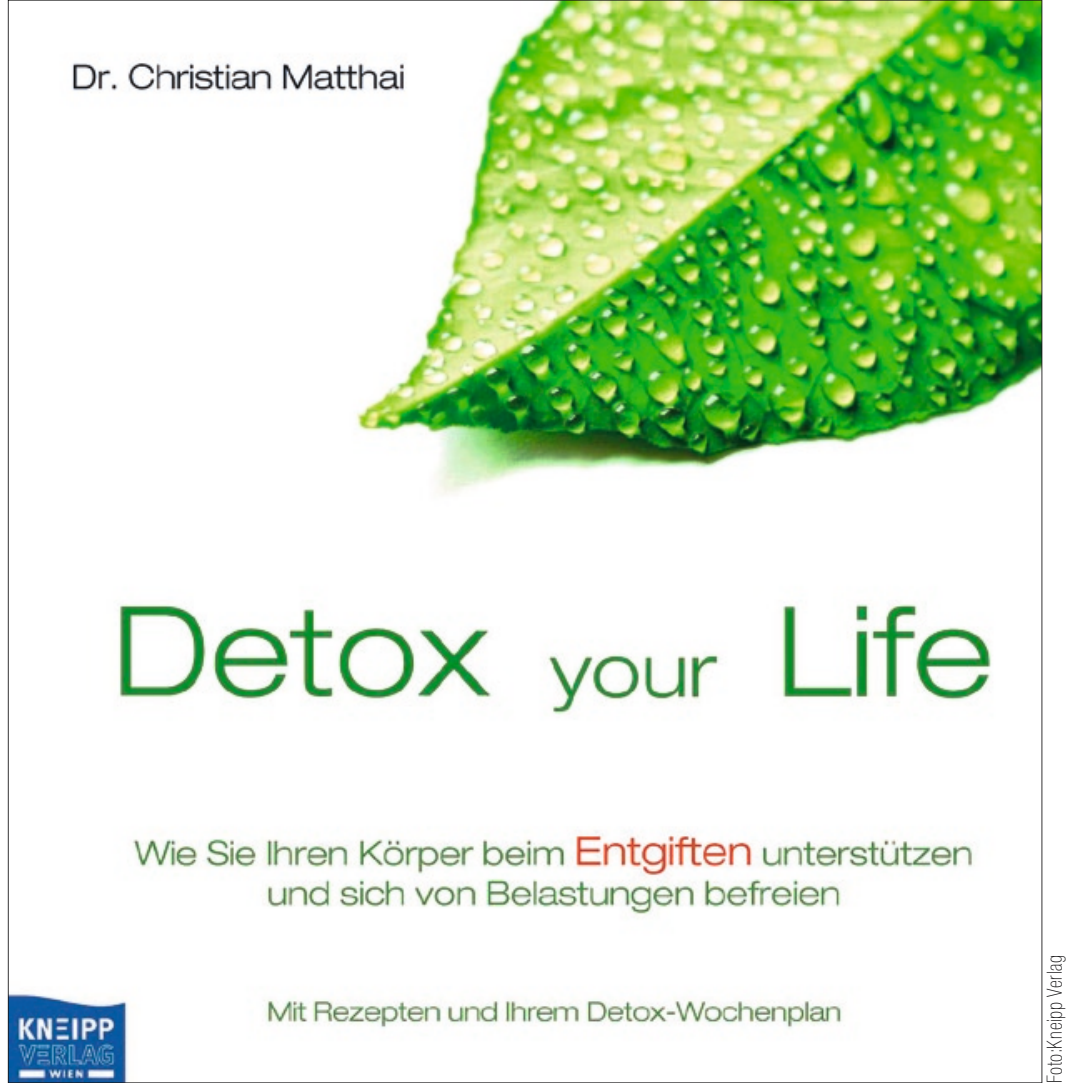

